\title{
THE ADVANTAGES OF PUBLIC AUCTIONS FOR COMPANIES IN FORESTRY
}

\author{
Slađana Đurić \\ Faculty of Economics in Subotica, University of Novi Sad, Republic of Serbia \\ dalisla@mts.rs
}

Original Scientific Paper doi:10.5937/jouproman7-23210

\begin{abstract}
JP "Vojvodinašume" Petrovaradin, Šumsko gazdinstvo „Sremska Mitrovica“ Sremska Mitrovica isprimary engaged in activities of management, maintenance, regeneration and protection of forests, as the natural resource, but also performs activities related to exploitation and utilization of forests. The Company finances its activities independently by selling its own timber assortments and services that are the result of its management performance. Timber assortments are sold through regular contracts for industrial buyers, independent contracts for companies and citizens, and in public auctions. This paperwork is to inform the public about timber auctions in this Company and to popularize this method of sales of timber assortments, as the method of selling product at prices higher than prices defined in actual price-list.
\end{abstract}

Key words: auctions, timber auctions, public auctions, industrial buyer, bidder

\section{Introduction}

The exploitation of forests, under the governance and management of JP "Vojvodinašume" Petrovaradin, Šumsko gazdinstvo „Sremska Mitrovica“ Sremska Mitrovica (The Public Company for Forest Management "Vojvodinašume" Petrovaradin, Forest holding ,Sremska Mitrovica“ Sremska Mitrovica) is performed within the limits of sustainable development and strict and comprehensive binding legal regulation in Serbia and according to International Conventions and Agreements.

The production of timber assortments is the outcome of performing necessary cultivation activities and rational exploitation of natural habitat potentials and economically represents the most important basic activity of the Company. The production range is estimated by 10 year Governance plans that specify the modes, amounts, structures, time periods and methods of production, according to defined overall goals of forest governance (JP "Vojvodinašume" Petrovaradin, 2016).

The management, maintenance, cultivation, regeneration and protection of Vojvodina forests is conducted with extremely high degree of responsibility and devotion. Exploitation and utilisation of forest is the activity carried out systematically and in accordance with long termplans, and production of timber assortments comes as a result of this activities. Timber assortments are usually sold to industrial buyers through annual contracts, to citizens and companies through independent transactions and by public auctions. Timber assortments for sale are mostly technical roundwood for further processing (industrial buyers/) and firewood (companies and citizens). Annual contracts for industrial buyers are prepared and determined in the main Directorate of JP "Vojvodinašume" Petrovaradin and quantities contracted are usually less than demands and needs of industrial buyers and, therefore, auctions are excellent opportunity for those buyers to purchase extra quantities of resources necessary for their further production. 
The sale of technical roundwood produces significant portion of incomes for the Company besides the sales of other timber assortments and services.

The JP"Vojvodinašume" Petrovaradin is consisted of following working units: Šumsko gazdinstvo (Forest holding),,Sremska Mitrovica“ Sremska Mitrovica, Šumsko gazdinstvo „Banat“" Pančevo, Šumsko gazdinstvo „Sombor“ Sombor, Šumsko gazdinstvo „Novi Sad“ Novi Sad, "Vojvodinašume- Lovoturs" Petrovaradin and "Vojvodinašume- Turist" Petrovaradin. Šumsko gazdinstvo „Sremska Mitrovica“ Sremska Mitrovica consists of five units: Šumska uprava (Forest administration) „Morović“, Šumska uprava „Višnjićevo“, Šumska uprava „Klenak“, Šumska uprava „Kupinovo“ and Forest mechanization.

This paperwork describes the procedures and results of actual timber auctions organized and conducted by Šumsko gazdinstvo „Sremska Mitrovica“ Sremska Mitrovica.

\section{Literature review}

"An auction is a market institution with explicit set of rules determining resource allocation and prices on the basis of bids from the market participants" (McAfee, McMillian, 1987)

Auctions are sales of assets, scheduled and organized in advance, to bidders who compete for offered assets according to their own estimation of assets value (Đurić, 2019).

„Auctions are an example of formal process for allocating and pricing the natural resources. They have generated research interest in economics, marketing and consumer behaviour fields. Auction maximizes the revenue for the seller, while being transparent and competitive method of allocation. An efficient auction design can achieve both an efficient assignment of rights to bidders and maximises revenue for the seller" (Frayet et al, 2013)

„Four basic types of auctions are used when a unique item is to be bought or sold: the English auction (open, oral, ascending bid auction), the Dutch (descending bid auction), the First price sealed bid auction and the Second price sealed bid auction (or Vickrey auction)“ (McAfee, McMillian, 1987)

English and Dutch auctions are the most used open auctions. In English auction the auctioneer starts the auction with his determined price, and bidders rise their bids until only one bidder remains (Klemperer, 2005).

The price can be increased by the seller of assets, the auctioneer (engaged by the seller or the auction Institution) or by bidders who participate in particular auction. The assets are sold to the bidder at the price he offered.

The Dutch auction starts with the auctioneer announcing very high price. The auctioneer than decreases the price and the first bidder who declares his readiness to buy assets at the announced price becomes the winner of the auction (Chakravarti et al, 2002).

In English and Dutch auction every bidder is aware of other bidders estimated values for assets offered for sale.

Bidders who participate in closed auctions deliver their offers in sealed envelopes and are not informed about other bidder's estimation of value for offered assets. 
The winner of the First price sealed bid auction is the participant who bids the higher price and that is the price he must actually pay in order to buy the asset he bids for. In the Second price sealed bid auction the winner is the bidder who submits the higher price, but to become the owner of the assets he bids for, he pays the amount of the second highest bid (Klemperer, 2005).

The auction process contains three main elements: auction issues, auction protocols and auction strategies. The bidders apply the auction protocols to express clear rules and procedures. These rules are used to send bids, accept or reject proposals, as well as to decide when the auction starts and ends. The bidder's preference and the need of the bidder at the time of auction are part of the auction strategies" (McAfee, McMillian, 1987).

\section{Auctions of timber roundwood}

We define state timber sale programs as the collection of laws, rules and operational policies, combined with the personnel and technology used to implement this laws, rules and policies that impact a state's ability to sell timber from the land it administers and manages (Brown et al, 2012)

In order to obtain the best possible outcome for the seller and the bidder, the auction process must be carried out by performing following sequence of steps (Đurić, 2019):

- Preparation of the auction: preparing all necessary paperwork, Decisions, Public call for participation in auction, forming Auction commission to monitor and evaluate the process of auction, setting the time and location of the auction, setting rules and procedures etc.;

- Setting the timber assortment and composing specific lots available for sale according to the timber type, category, quality and setting the price;

- Placing timber lots at the location specified in the Public call for bidders and enabling bidders to inspect and check lots prior to taking place in auction;

- Providing bidders with all necessary information related to the lot set for auction, terms and procedures of the auction, payment rules etc.;

- Conducting the auction, according to the chosen type and in accordance with all rules for that type of the auction;

- Selling the object of the auction to winning bidder, performing all procedures related to the sale, providing bidders with the Record/report on auction and submission of all necessary documents related to terminated auction;

- Ownership transfer, according to defined methods, signing Contracts, payment etc.

English auction is used for sale of technical roundwood in JP "Vojvodinašume" Petrovaradin, Šumsko gazdinstvo "Sremska Mitrovica“ Sremska Mitrovica.

The preparation of timber assortments for sale consists of following activities (Ranković, Keča, 2011): 
- Harvesting and processing of timber assortments, measuring, classification and branding logs (with hammer or by placing a plastic plate with forest mark);

- Pulling logs and transporting them to selling location;

- Assorting and organizing logs in lots;

- Pricing timber assortments;

- Specifying terms and procedures of sale.

Technical roundwood in JP "Vojvodinašume" Petrovaradin, Šumsko gazdinstvo "Sremska Mitrovica" Sremska Mitrovica is sold at forest haul road in manner ,seen-bought".

\section{Auctions in Šumsko gazdinstvo „Sremska Mitrovica“ Sremska Mitrovica}

Following hypothesis were set in this research:

H1: The auction enables the Company to sell the surplus of technical roundwood to buyers who are interested to purchase those products

$\mathrm{H} 2$ : The public auction enables the buyers to purchase quantity of necessary technical roundwood that exceed quantities determined in annual contract

H3: Selling technical roundwood in auctions enables the Company to sell its products at higher prices than prices established in regular price-list for the business period in which the auction took place

H4: The number of bidders that participate in auction significantly affects the price reached in auction
Picture: FSC sign used in JP "Vojvodinašume" Petrovaradin, Šumsko gazdinstvo "Sremska Mitrovica“ Sremska Mitrovica

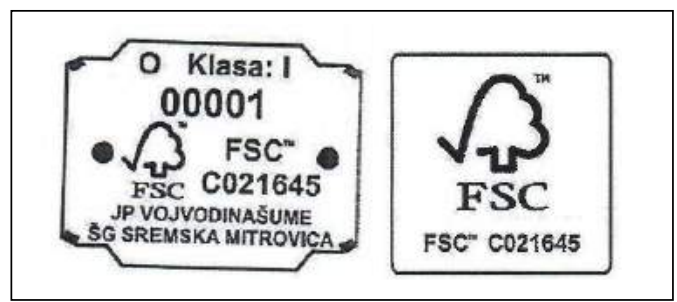

All timber assortments produced in JP "Vojvodinašume" Petrovaradin, Šumsko gazdinstvo "Sremska Mitrovica" Sremska Mitrovica are marked with FCS sign as the guarantee of timber origin and quality.

Commercial sector submits the Request for auction of timber assortments based on their Realization reports and the fact that there is surplus of products that might be sold at public auction.Director of JP "Vojvodinašume" Petrovaradin approves the request from Commercial sector and issues Decision for organizing public auction for sale of technical roundwood.Pricing strategy of this Company is based on market principles of offer and demand and is adjusted to legal regulations. Lot prices are derived from actual Price-list for that particular year for the type, class, quality and quantity of specific assortments. Starting price for every lot offered for auction sale is specified in Public contest for auction of technical roundwood (announced at the official web page of JP "Vojvodinašume" Petrovaradin). This Public contest contains all relevant information related to lots specification, location for auction, which Forest administration is in charge, starting prices and contact personnel. The Application form for participation in public auction may be obtained from Forest administrations and downloaded from official web page of the Company. 
Terms and procedures for participation in timber assortments public auctions may also be obtained from official web page of the Company. Members of Auction commission are appointed by Representative/agent of Šumsko gazdinstvo "Sremska Mitrovica" Sremska Mitrovica (former position: Director) and are responsible for entire auction process including issuing Record/report on terminated auction. This Record/report containing all information of particular auction (time started/ended, location, lot details, prices, number of participants, the list of participants with the amounts of advance payments for participation in auction, actual bidding process, the winner and all other relevant information)is delivered to all participants after the auction is terminated.

\begin{tabular}{|c|c|c|c|c|c|c|}
\hline Assortment & Quantity & $\%$ & Value in dinars & $\%$ & Average price & $\%$ \\
\hline Oak F & $2.980,67$ & 163,41 & $147.954 .036,64$ & 321,41 & $49.637,78$ & 196,68 \\
\hline Ash F & 200,90 & 38,12 & $6.714,025,63$ & 78,81 & $33.419,74$ & 206,73 \\
\hline OTLF & 87,84 & & $3.541 .017,06$ & & & \\
\hline Poplar F/L & $43.495,05$ & 122,94 & $349.430 .936,13$ & 136,24 & $8.033,81$ & 110,81 \\
\hline Oak P & $17.530,40$ & 108,63 & $230.915 .394,48$ & 116,86 & $13.172,28$ & 107,58 \\
\hline Ash P & $3.580,38$ & 92,76 & $39.620 .653,74$ & 93,92 & $11.066,05$ & 101,25 \\
\hline OTL P & $1.953,95$ & 151,70 & $16.305 .388,62$ & 168,97 & $8.344,83$ & 111,38 \\
\hline Poplar P & $17.300,93$ & 111,38 & 79.131.726,97 & 127,93 & $4.573,84$ & 114,85 \\
\hline OML P & $2.006,30$ & 120,50 & $8.197 .515,33$ & 126,77 & $4.085,89$ & 105,21 \\
\hline Small roundwood & 610,05 & 48,26 & $3.319 .285,43$ & 52,20 & $5.441,01$ & 108,15 \\
\hline Total roundwood & $89.746,47$ & 115,84 & $885.129 .980,03$ & 139,36 & $9.862,56$ & 120,31 \\
\hline Technical logs & $8.428,05$ & 73,21 & $28.856 .539,59$ & 93,12 & $3.423,87$ & 127,19 \\
\hline Pulpwood, wood for panels, firewood ML & $9.501,09$ & 93,53 & $22.676 .041,18$ & 82,99 & $2.386,68$ & 88,73 \\
\hline Firewood TL & $56.962,42$ & 65,07 & $235.824 .779,31$ & 74,27 & $4.140,01$ & 114,14 \\
\hline Totaltimber assortment: & $164.638,04$ & 88,19 & 1.172.487.340,11 & 115,98 & $7.121,61$ & 131,50 \\
\hline
\end{tabular}

Source: Internal documents of JP „Vojvodinašume“ Petrovaradin, Šumsko gazdinstvo“Sremska Mitrovica“ Sremska Mitrovica

Legend: F-logs for veneer, P-sawmill log, L-peeler log, TL-hardwood, OTL-the rest hardwood, ML-softwood, $O M L$ - the rest softwood

This table is given with all details in order to present the full list of timber assortments produced in Šumsko gazdinstvo"Sremska Mitrovica" Sremska Mitrovica (this part of paperwork presents auctions conducted in this working unit of JP "Vojvodinašume" Petrovaradin).

Table 2: Timber assortment realization: 2018- June 2019

\begin{tabular}{|c|c|c|c|c|c|c|}
\hline \multicolumn{7}{|c|}{ January-December 2018} \\
\hline Assortment & Quantity & $\%$ & Value in dinars & $\%$ & Average price & $\%$ \\
\hline Total roundwood & $94.471,41$ & 121,95 & $915.143 .725,56$ & 121,62 & $9.686,99$ & 99,73 \\
\hline $\begin{array}{l}\text { Total timber } \\
\text { assortment: }\end{array}$ & $165.384,34$ & 88,69 & $1.181 .849 .338,19$ & 100,15 & $7.146,08$ & 112,91 \\
\hline \multicolumn{7}{|c|}{ January-June 2019} \\
\hline Assortment & Quantity & $\%$ & Value in dinars & $\%$ & Average price & $\%$ \\
\hline Total roundwood & $47.628,22$ & 62,20 & $537.334 .745,66$ & 71,11 & $11.281,86$ & 114,33 \\
\hline $\begin{array}{l}\text { Total timber } \\
\text { assortment }\end{array}$ & $79.494,71$ & 42,28 & $657.961 .465,58$ & 53,57 & $8.276,88$ & 126,69 \\
\hline
\end{tabular}

Realisation for period 2018-June 2019 is presented in simple table containing only figures for „Total roundwood“" and „Total timber assortments“. 


\begin{tabular}{|c|c|c|c|c|c|c|}
\hline \multicolumn{7}{|c|}{ Table 3: Planned production of timber assortments for period 2017-2019 } \\
\hline \multicolumn{7}{|c|}{2017} \\
\hline Assortment & Quantity & \multicolumn{3}{|c|}{ Value in dinars } & \multicolumn{2}{|c|}{ Average price in dinars } \\
\hline Total roundwood & & 77.477 & \multicolumn{2}{|r|}{635.149 .566} & \multicolumn{2}{|r|}{8.198} \\
\hline Total timber assortment & & 186.682 & & 1.010 .973 .946 & & 5.415 \\
\hline \multicolumn{7}{|c|}{2018} \\
\hline Assortment & Quantity & \multicolumn{3}{|c|}{ Value in dinars } & \multicolumn{2}{|c|}{ Average price in dinars } \\
\hline Total roundwood & & \multicolumn{2}{|l|}{77.469} & 752.484 .614 & \\
\hline Total & & \multicolumn{2}{|l|}{186.465} & \multirow[t]{2}{*}{ 1.180.127.453 } & \multicolumn{2}{|r|}{6.329} \\
\hline \multicolumn{6}{|c|}{2019} & \\
\hline Assortment & Quantity & \multicolumn{3}{|c|}{ Value in dinars } & \multicolumn{2}{|c|}{ Average price in dinars } \\
\hline \multirow{2}{*}{\multicolumn{2}{|c|}{$\begin{array}{l}\text { Total roundwood } \\
\text { Total timber assortment }\end{array}$}} & \multicolumn{2}{|l|}{76.579} & 755.652 .013 & \multicolumn{2}{|r|}{9.868} \\
\hline & & \multicolumn{2}{|l|}{188.020} & 1.228 .338 .938 & \multicolumn{2}{|r|}{6.533} \\
\hline \multicolumn{7}{|c|}{$\begin{array}{l}\text { Source: Internal documents of JP „Vojvodinašume“ Petrovaradin, Šumsko gazdinstvo“Sremska Mitrovica“ Sremska } \\
\text { Mitrovica }\end{array}$} \\
\hline \multicolumn{7}{|c|}{$\begin{array}{l}\text { Data in tables presented above demonstrate that for categories „Total roundwood" and "Total timber assortment" } \\
\text { Sumsko gazdinstvo"Sremska Mitrovica" Sremska Mitrovica reached and exceeded planned production quotas } \\
\text { and that surplus of products might be sold in public auctions to buyers who are interested in purchasing these } \\
\text { products for their production activities. }\end{array}$} \\
\hline Table & 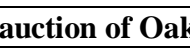 & & & & & \\
\hline Forest & 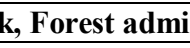 & ion Viš & 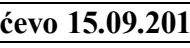 & & & \\
\hline Party & 1 & 2 & 3 & 4 & 5 & Total \\
\hline Buyer & Buyer 1 & Buyer 2 & Buyer 3 & Buyer 4 & Buyer 5 & \\
\hline Assortment & Oak sawlog & Oak sawlog & Oak sawlog & Oak sawlog & Oak sawlog & \\
\hline Quantity in $\mathbf{m}^{3}$ & 44,94 & 45,27 & 45,16 & 45,03 & 53,36 & 225,76 \\
\hline Starting price for $\mathbf{m}^{3}$ & $13.760,00$ & $13.057,00$ & $13.767,00$ & $12.563,00$ & $12.027,00$ & \\
\hline lue in dinars & $618.374,40$ & $591.090,39$ & $621.717,72$ & $565.711,89$ & $545.544,72$ & 2.942.439,12 \\
\hline Achieved price $\operatorname{din} / \mathbf{m}^{3}$ & $28.000,00$ & $28.000,00$ & $33.500,00$ & $22.500,00$ & $20.000,00$ & \\
\hline Total achieved value in din & $1.258 .320,00$ & $1.267 .560,00$ & $1.512 .860,00$ & $1.013 .175,00$ & $907.200,00$ & $5.959 .115,00$ \\
\hline More than & $639.945,60$ & $676.469,61$ & $891.142,28$ & $447.463,11$ & $361.655,28$ & 3.016.675,88 \\
\hline & 103,49 & 114,44 & & 79.10 & 66,29 & 102,52 \\
\hline in din & $1.509 .984,00$ & $1.521 .072,00$ & $1.815 .432,00$ & $1.215 .810,00$ & $1.088 .640,00$ & $7.150 .938,00$ \\
\hline Payed in advance din & $295.000,00$ & 0,00 & $294.245,00$ & $111.125,96$ & $294.249,91$ & $994.614,87$ \\
\hline Total for payment in din & $1.214 .984,00$ & $1.521 .072,00$ & $1.521 .187,00$ & $1.104 .684,04$ & $794.396,09$ & 6.156.323,13 \\
\hline
\end{tabular}

Table above presents final report on auction held in Šumska Uprava Klenak and Šumska uprava Višnjićevo on 15.09.2017.

Table 5: The final report on auction of Oak and Ash veneer logs

\begin{tabular}{|c|c|c|c|c|c|c|}
\hline \multicolumn{7}{|c|}{ Forest administration Morović 06.02.2018. } \\
\hline Party & 1 & 2 & 3 & 4 & 5 & Total \\
\hline Buyer & Buyer 1 & Buyer 2 & Buyer 3 & Buyer 4 & Buyer 5 & \\
\hline Assortment & $\begin{array}{l}\text { Oak veneer } \\
\quad \log \end{array}$ & $\begin{array}{l}\text { Oak veneer } \\
\log \end{array}$ & $\begin{array}{l}\text { Oak veneer } \\
\quad \log \end{array}$ & $\begin{array}{l}\text { Oak veneer } \\
\log \end{array}$ & $\begin{array}{l}\text { Ash veneer } \\
\quad \log \end{array}$ & \\
\hline Quantity in $\mathbf{m}^{3}$ & 43,21 & 42,68 & 43,26 & 42,19 & 48,22 & 219,56 \\
\hline Starting price for $\mathbf{m}^{3}$ & $63.670,00$ & $62.970,00$ & $63.250,00$ & $38.870,00$ & $33.980,00$ & \\
\hline Starting value in din & $2.751 .180,70$ & $2.687 .559,60$ & $2.736 .196,00$ & $1.639 .925,30$ & $1.638 .516,60$ & 11.453.378,20 \\
\hline $\begin{array}{l}\text { Achieved price din/ } \\
\text { m }^{3}\end{array}$ & $125.000,00$ & $111.000,00$ & $129.000,00$ & $87.000,00$ & $38.980,00$ & \\
\hline $\begin{array}{l}\text { Total achieved value } \\
\text { in din }\end{array}$ & $5.401 .250,00$ & 4.737.480,00 & $5.580 .540,00$ & $3.670 .530,00$ & $1.879 .615,60$ & $21.269 .415,60$ \\
\hline \multirow{2}{*}{$\begin{array}{l}\text { More than starting } \\
\text { price din } / \%\end{array}$} & $2.650 .069,30$ & $2.049 .920,40$ & $2.844 .344,0$ & $2.030 .604,70$ & $241.099,00$ & $9.816 .037,40$ \\
\hline & 96,32 & 76,27 & 103,95 & 123,82 & 14,71 & 85,70 \\
\hline $\begin{array}{l}\text { Total for payment, } \\
\text { VAT included }\end{array}$ & $6.481 .500,00$ & $5.684 .976,00$ & $6.696 .648,00$ & $4.404 .636,00$ & $2.255 .538,72$ & $25.523 .298,72$ \\
\hline $\begin{array}{l}\text { Source: Internal doct } \\
\text { Mitrovica }\end{array}$ & s of $\mathrm{JP}, \mathrm{V}$ & inašume“" & aradin,, S & o gazdinst & mska Mit & ca"Sremska \\
\hline
\end{tabular}




\begin{tabular}{|c|c|c|c|c|c|c|c|}
\hline orest administration Mo & Iorović 30.11.20 & & & & & & \\
\hline Party & 1 & 2 & & 3 & 4 & 5 & Total \\
\hline Buyer & Buyer 1 & Buye & & Buyer 3 & Buyer 4 & Buyer 5 & \\
\hline Assortment & $\begin{array}{l}\text { ak and cherry } \\
\text { sawmill logs }\end{array}$ & $\begin{array}{r}\text { Oak ve } \\
\log \end{array}$ & & $\begin{array}{l}\text { Oak veneer } \\
\qquad \log \end{array}$ & $\begin{array}{l}\text { Oak veneer } \\
\qquad \log \end{array}$ & $\begin{array}{l}\text { Oak veneer } \\
\qquad \log \end{array}$ & \\
\hline Quantity in $\mathbf{m}^{3}$ & 46,30 & &, 42 & 47,04 & 49,39 & 71,84 & 258,99 \\
\hline $\begin{array}{l}\text { Starting price for } \\
\mathbf{m}^{3}\end{array}$ & $34.430,00$ & 42.0 &, 00 & $36.290,00$ & $36.290,00$ & $33.370,00$ & \\
\hline $\begin{array}{l}\text { Starting value in } \\
\text { din }\end{array}$ & $1.594 .109,00$ & 1.869 .6 &, 80 & $1.707 .081,60$ & $1.792 .363,10$ & 2.397.300,80 & $9.360 .492,30$ \\
\hline $\begin{array}{l}\text { Achieved price } \\
\operatorname{din} / \mathbf{m}^{3}\end{array}$ & $56.500,00$ & 61.0 &, 00 & $49.000,00$ & $46.500,00$ & $64.000,00$ & \\
\hline $\begin{array}{l}\text { Total achieved } \\
\text { value din }\end{array}$ & $2.615 .950,00$ & 2.709 .6 &, 00 & $2.304 .960,00$ & $2.296 .635,00$ & $4.597 .760,00$ & $14.524 .925,00$ \\
\hline $\begin{array}{l}\text { More than } \\
\text { starting price }\end{array}$ & $1.021 .841,00$ & 839.9 & 20 & $597.878,40$ & $504.271,90$ & $2.200 .459,20$ & $5.164 .432,70$ \\
\hline $\operatorname{din} / \%$ & 64,10 & & 1,93 & 35,02 & 28,13 & 91,79 & 55,17 \\
\hline $\begin{array}{l}\text { Total, VAT } \\
\text { included }\end{array}$ & $3.139 .140,00$ & 3.251 .5 & 1,00 & $2.765 .952,00$ & $2.755 .962,00$ & $5.517 .312,00$ & 17.429,910,00 \\
\hline $\begin{array}{l}\text { Payed in advance } \\
\text { din }\end{array}$ & $159.410,90$ & 346.3 & 1,68 & $569.849,14$ & $776.638,33$ & $936.049,23$ & 2.788.322,28 \\
\hline $\begin{array}{l}\text { Total for payment } \\
\text { in din }\end{array}$ & $2.979 .729,10$ & 2.905 .1 &, 32 & $2.196 .102,86$ & $1.979 .323,67$ & $4.581 .262,77$ & 14.641.587,72 \\
\hline $\begin{array}{l}\text { Source: Internal documen } \\
\text { Mitrovica } \\
\end{array}$ & ents of JP, „Vojv & dinašu & 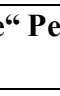 & rovaradin, Šun & $\begin{array}{l}\text { msko gazdinstvo } \\
\end{array}$ & "Sremska Mitro & ica"s Sremska \\
\hline Table above presents & ts final repo & nas & & in Šum & ska Uprava $\mathrm{N}$ & Morović on 30 & 11.2018 \\
\hline $\begin{array}{l}\text { Table 7: The final report } \\
\text { Forest administration Mo } \\
\end{array}$ & $\begin{array}{l}t \text { on auction of } \mathrm{O} \\
\text { orović 14.12.201 }\end{array}$ & 3. & & & & & \\
\hline Party & 1 & & & 2 & 3 & 4 & Total \\
\hline Buyer & Buy & $\mathbf{r} 1$ & & yer 2 & Buyer 3 & Buyer 4 & \\
\hline Assortment & Oak ve & her $\log$ & Oak & vener log & ak vener $\log$ & Oak vener log & \\
\hline Quantity in $\mathbf{m}^{3}$ & & 45,29 & & 45,89 & 44,89 & 41,48 & $\mathbf{1 7 7 , 5 5}$ \\
\hline Starting price for $\mathbf{m}^{3}$ & & 795,00 & & $44.750,00$ & $45.860,00$ & $51.800,00$ & \\
\hline Starting value in din & 2.300 & 505,55 & & $53.577,50$ & $2.063 .144,40$ & $2.148 .664,00$ & $8.565 .891,45$ \\
\hline Achieved price in $\operatorname{din} / \mathrm{m}^{3}$ & & 800,00 & & $48.750,00$ & $48.000,00$ & $54.300,00$ & \\
\hline Achieved value in din & 2.527 & 182,00 & & $37.137,50$ & $2.154 .720,00$ & $2.252 .364,00$ & 9.171.403,50 \\
\hline $\begin{array}{l}\text { More than starting price } \\
\text { din/\% }\end{array}$ & 226 & 676,45 & & $33.560,00$ & $91.575,60$ & $103.700,00$ & $605.512,05$ \\
\hline & & 9,85 & & 8,94 & 4,44 & 4,83 & 7,07 \\
\hline Total, VAT included & 3.032 & 618,40 & & $34.565,00$ & $2.585 .664,00$ & $2.702 .836,80$ & 11.005.684,20 \\
\hline Payed in advance din & 230 & 050,56 & & $11.672,19$ & $641.722,74$ & $626.538,59$ & $1.909 .984,08$ \\
\hline Total for payment in din & 2.802 & 567,84 & & $72.892,81$ & $1.943 .941,26$ & $2.076 .298,21$ & $9.095 .700,12$ \\
\hline
\end{tabular}

Source: Internal documents of JP „Vojvodinašume“ Petrovaradin, Šumsko gazdinstvo“Sremska Mitrovica“ Sremska Mitrovica

Table above presents final report on auction held in Šumska Uprava Morović on 14.12.2018. 


\begin{tabular}{|c|c|c|c|c|c|c|}
\hline \multicolumn{7}{|c|}{ Forest administration Kupinovo 12.02.2019. } \\
\hline Party & 1 & 2 & 3 & 4 & 5 & Total \\
\hline Buyer & Buyer 1 & Buyer 2 & Buyer 3 & Buyer 4 & Buyer 5 & \\
\hline Assorment & $\begin{array}{l}\text { Oak veneer } \\
\qquad \log \end{array}$ & $\begin{array}{l}\text { Oak veneer } \\
\log \end{array}$ & $\begin{array}{l}\text { Oak veneer } \\
\log \end{array}$ & $\begin{array}{l}\text { Oak veneer } \\
\log \end{array}$ & $\begin{array}{l}\text { Oak veneer } \\
\log \end{array}$ & \\
\hline Quantity in $\mathbf{m}^{3}$ & 44,141 & 44,25 & 44,38 & 44,48 & 65,55 & 242,80 \\
\hline $\begin{array}{l}\text { Starting price for } \\
\mathbf{m}^{3}\end{array}$ & $53.970,00$ & $53.700,00$ & $50.580,00$ & $53.140,00$ & $53.370,00$ & \\
\hline $\begin{array}{l}\text { Starting price in } \\
\text { din }\end{array}$ & $2.382 .235,80$ & $2.376 .225,00$ & $2.244 .740,40$ & 2.363.337,20 & $3.498 .403,50$ & 12.865.271,90 \\
\hline $\begin{array}{l}\text { Achieved price din/ } \\
\text { m }^{3}\end{array}$ & $57.000,00$ & $56.200,00$ & $53.080,00$ & $60.640,00$ & $55.870,00$ & \\
\hline $\begin{array}{l}\text { Total achieved } \\
\text { value din }\end{array}$ & $2.515 .980,00$ & $2.486 .850,00$ & $2.355 .690,40$ & 2.697.267,20 & $3.662 .278,50$ & 13.718.066,10 \\
\hline \multirow{2}{*}{$\begin{array}{l}\text { More than starting } \\
\text { price } \\
\text { din/\% }\end{array}$} & $\begin{array}{r}133.744,20 \\
\operatorname{din}\end{array}$ & $\begin{array}{r}110.625,00 \\
\operatorname{din}\end{array}$ & $\begin{array}{r}110.950,00 \\
\operatorname{din}\end{array}$ & $\begin{array}{r}333.600,00 \\
\text { din }\end{array}$ & $\begin{array}{r}163.875,00 \\
\operatorname{din}\end{array}$ & $\begin{array}{r}852.794,20 \\
\text { din }\end{array}$ \\
\hline & $5,61 \%$ & 4,66 & 4,94 & 14,11 & 4,68 & 6,63 \\
\hline $\begin{array}{l}\text { Total, VAT } \\
\text { included }\end{array}$ & $3.019 .176,00$ & $2.984 .220,00$ & $2.826 .828,48$ & $3.236 .720,64$ & $4.394 .734,20$ & 16.461.679,32 \\
\hline Payed in advance & $474.590,30$ & $237.622,50$ & $224.474,04$ & $810.681,11$ & $824.430,65$ & $2.571 .798,60$ \\
\hline $\begin{array}{l}\text { Total for payment } \\
\text { in din }\end{array}$ & $2.544 .585,70$ & $2.746 .597,50$ & $2.602 .354,44$ & $2.426 .039,53$ & $3.570 .303,55$ & $13.889 .880,72$ \\
\hline
\end{tabular}

Table above presents final report on auction held in Šumska Uprava Kupinovo on 12.02.2019.

Comparison of tables of „Timber assortment realization“ $(1,2)$ „Planned production of timber assortments" (3) and

„Final report on held auctions“" $(4,5,6,7,8)$ suport claims $\mathrm{H} 1, \mathrm{H} 2$ and $\mathrm{H} 3$.

H1: The auction enables the Company to sell the surplus of technical roundwood to buyers who are interested to purchase those products

$\mathrm{H} 2$ : The public auction enables the buyers to purchase quantity of necessary technical roundwood that exceed quantities determined in annual contract

H3: Selling technical roundwood in auctions enables the Company to sell its products at higher prices than prices established in regular price-list for the business period in which the auction took place

Data given in „Timber assortment realization" $(1,2)$ show that actual production of timber assortments exceed planned production quantities, demonstrated in „Planned production of timber assortments" (3) and that surplus of produced assortments are available for sale to interested buyers. Auction sale of these products satisfies both, Šumsko gazdinstvo"Sremska Mitrovica" Sremska Mitrovica, which is in the position to sell its products at prices higher than those in Price-list, and buyers who are interested to buy assortment for their further production.

Data demonstrated in tables „Final report on held auctions“ $(4,5,6,7,8)$ provide evidence that prices achieved in public auction of technical roundwood in period 2017-June 2019 are significantly higher than regular prices derived from actual Price-list. 
H4: The number of bidders that participate in auction significantly affects the price reached in auction

Auction held on 15.09.2017. had 10 bidders who competed for 5 lots of technical roundwood. Prices achieved in this particular auction are in average $102,52 \%$ higher than starting prices. Other auctions had 4-7 bidders competing for 4-5 lots of technical roundwood, and prices achieved in these auctions are lower than in auction with 10 participants which proves $\mathrm{H} 4$ to be valid.

\section{Discussion}

Responsible and sustainable management of forest and planned and systematically performed activities of its exploitation and utilisation in this Company produces significant surplus of products available for sale in public auctions.

The quality of timber assortment, that composes lots presented for sale, significantly affects the price achieved in the auction.

Managing and governing natural resource in responsible and law obeying manner, caused JP "Vojvodinašume" Petrovaradin, Šumsko gazdinstvo "Sremska Mitrovica" Sremska Mitrovica, to achieve and exceed planned norms and predicted production levels in observed period of time (but it is a long term determination of the Company's management and workers) and produced significant amount of technical roundwood, disposable for auction selling. Selling extra amounts of technical roundwood in public auction gives the opportunity to the Company to allocate its products by reaching significantly higher prices than those determined in the regular Price -list, and to obtain additional sale income. For buyers, these auctions are convenient since they are in the position to purchase extra amount of resources necessary for their industrial production.

The average (starting price) price for particular lot is derived from the actual Price-list for different type, class and quality of wood (in that particular lot), according to its share in that lot. The auction process proved that prices achieved at the end of auction might be significantly higher than those in actual Price-list (even more than $100 \%$ higher) which is the great opportunity for the Company to increase its revenue from sale.

„One more bidder than the number of prizes is not enough in an ascending auction, even in a one-off auction in which the bidder can win at most one prize each (so there is no question of colluding to divide prizes“(Klemperer, 2005).

Number of participants is very significant element of the auction and affects the final prices achieved in the auction. Demonstrated auction reports revealed that significantly higher prices are obtained in auctions with 10 bidders, than in those auctions with 4 or 5 bidders.

It is important to mention that the quality of timber products consisting one particular lot for sale, determine the prices achieved in auctions as well. Lots are formed of the wood of similar quality and characteristics, but not all products containing one lot match demands of all potential buyers. Needs and drives of bidders are determined by their production capacities and characteristics. Therefore, the composition of particular lots must be adjusted to buyer's needs. If the timber harvested and prepared for sale is not of satisfying quality, the price achieved in the auction might be under desired limits. 
Technical roundwood sold in auction held in 2017. was of higher quality than the quality of technical roundwood sold in later auctions, and that might have affected achieved prices, even though the quality of technical roundwood and all other timber products coming from forests governed by JP "Vojvodinašume" Petrovaradin, Šumsko gazdinstvo "Sremska Mitrovica" Sremska Mitrovica are of very high quality.

Prices achieved in the auction are determined by the market request. Demand for Ash tree was higher than the offer in period 2017-2019, and that resulted in lower interest and lower prices achieved.

Therefore, in order to finish auction process with satisfying results, it is of vital importance to follow offer-demand principles of the market and to respect needs of the bidders in terms of quantity and quality of offered lots.

According to the author, forming one team within the Company in charge of preparing and processing auctions might improve current auction practice in the Company. This unit should employ experts of Forestry, Finance, Marketing, Public relations and IT sector. This team would prepare necessary paperwork (this is currently done by Commercial sector), improve auction rules and procedures, follow the process of auction and complete all actions related to every singular auction. In addition to that, this team, would study auction practice in other countries with wide areas under the forests that organize public auctions frequently. In USA, Canada, Germany, Poland, Czech Republic, Slovakia and other countries with developed Forestry, public auctions are held even on monthly basis. These auctions differ from auctions in JP "Vojvodinašume" Petrovaradin, Šumsko gazdinstvo "Sremska Mitrovica" Sremska Mitrovica, not only in the amounts of technical roundwood disposable for auctions, but also in terms and manners they are conducted in. The object of the auction in these countries are standing trees offered for harvesting to companies with manpower, knowledge and equipment for harvesting trees in forests. In our country such companies are very few, and they are predominantly Public Companies for governing and managing forests and Public Companies governing National parks. Also, industrial buyers who buy products from JP "Vojvodinašume" Petrovaradin, Šumsko gazdinstvo "Sremska Mitrovica" Sremska Mitrovica do not possess necessary manpower, expertise and equipment to harvest standing trees themselves. Another distinction is the process of the auction. Auctions in these countries are conducted via Internet. Potential buyers are given catalogue, with exact forest sites for harvesting, characteristics of the trees, pictures of forest sites, annual schedule for auctioning, the overview of prices, procedures, deadlines, payment terms etc. Internet websites are regularly updated with eventual changes and consist all relevant data, Auction websites are interactive and enable all bidders to participate and place offers within the period of time determined for auction from distant locations.

Creating team in charge of auctions, preparing catalogue (3D and easy for download) for potential bidders (such catalogue does not exist in this moment), creating interactive auction website and aggressive promotion of auctions might improve auction process in JP "Vojvodinašume" Petrovaradin, Šumsko gazdinstvo "Sremska Mitrovica" Sremska Mitrovica. This improved approach in organizing auctions might lead auctions to become one of crucial methods of sale in this Company and might significantly increase its revenue from sale. 


\section{Conclusion}

The rise of e-commerce, government privatizations and both public and private outsourcing has greatly increased the role of auctions in the economy (Klemperer, 2005)

"Traditional auctioneers recognize the importance of marketing in their trade and discuss advertising principles and the "psychology of selling" as keys auctioneering success. The growing popularity of Internet auctions is also driving new product-market and pricing models, revised channel roles and new market research methods (Chakravarti et al, 2002).

Aggressive PR, professional personnel, interactive website, annual calendar of auction sales and more profound customer relationship approach might promote this Company into regional leader in innovations related to auction sales. Electronic auction sales would make regional cooperation easier and increase financial return from sale.

"The most important assumption of sustainable governance is the awareness that the utilisation and natural conditions are interdependent, and that temporary production processes must consider future needs"(Schmithüsen,2006).

\section{References}

Brown R.N., Kilgore M.A., Blinn C.R., Coggins J.S., (2012), State Timber Sale Programs, Policies and Procedures: A National Assessment, Journal of Forestry, Society of American Foresters

Chakravarti D., Greenleaf E., Sinha A., Cheema A., Cox J.C., Friedman D., Ho T.H., Isaac R.M., Mitchell A.A.,Rapoport A., Rothkopf M.H., Srivastava J., Zwick R., (2002), Auctions: Research opportunities in marketing, Marketing letters 13:3,281-296, Kluwer academic publishers, Netherlands
Đurić, S., (2019) Research paperwork: The auction of technical timber in The Public Company for Forest Management "Vojvodinašume" Petrovaradin, Forest holding „Sremska Mitrovica“ Sremska Mitrovica, University of Novi Sad, Faculty of Economics in Subotica

Frayret J-M, Farnia F., LeBel L., Beaudry C. (2013), Multiple-round timber auction design and sumulation, Cirrelt-2013-12, Interuniversity research centre on European networks, logistics and transportation, Quebec, Canada

JP "Vojvodinašume" Petrovaradin (2016), The Public Company for Forest Management "Vojvodinašume" Petrovaradin, The annual bussiness plan for 2017., www.vojvodinasume.rs, access june 2019

Klemperer P., (2005), Competition policy in auctions and „Bidding markets“, Nuffield College, Oxford OX1 1NF, England

McAfee, R.P., McMillan J., (1987), Auctions and bidding, Journal of economic literature, Vol.25, No.2, 699-738

Ranković N., Keča Lj., 2011, Trgovina i marketing šumskih proizvoda, Univerzitet u Beogradu, Šumarski fakultet, Beograd

Schmithüsen F., Kaiser B., Schmidhauser A., Mellinghoff S., Kammerhofer A.W., (2006), Preduzetništvo u šumarstvu i drvnoj industriji, Osnove menadžmenta i poslovanja, Centar za izdavačku delatnost Ekonomskog fakulteta $\mathrm{u}$ Beogradu 